\title{
Obstacle Crossing Differences Between Blind and Blindfolded Subjects After Haptic Exploration
}

\author{
Arturo Forner-Cordero, Valéria D. Garcia, Sérgio T. Rodrigues \& Jacques \\ Duysens
}

To cite this article: Arturo Forner-Cordero, Valéria D. Garcia, Sérgio T. Rodrigues \& Jacques Duysens (2016) Obstacle Crossing Differences Between Blind and Blindfolded Subjects After Haptic Exploration, Journal of Motor Behavior, 48:5, 468-478, DOI:

10.1080/00222895.2015.1134434

To link to this article: https://doi.org/10.1080/00222895.2015.1134434

册 Published online: 02 Jun 2016.

Submit your article to this journal

Џ Article views: 149

View Crossmark data \lceil

Citing articles: 1 View citing articles 
RESEARCH ARTICLE

\title{
Obstacle Crossing Differences Between Blind and Blindfolded Subjects After Haptic Exploration
}

\author{
Arturo Forner-Cordero', Valéria D. Garcia², Sérgio T. Rodrigues ${ }^{3}$, Jacques Duysens ${ }^{4}$ \\ ${ }^{1}$ Biomechatronics Lab. Mechatronics Department, Escola Politécnica da Universidade de São Paulo, São Paulo, Brazil. \\ ${ }^{2}$ Neuroscience and Behavior, Institute of Psychology, University of São Paulo, São Paulo, Brazil. ${ }^{3}$ Laboratory of Information, \\ Vision, and Action (LIVIA), UNESP—State University of São Paulo, Bauru, Brazil. ${ }^{4}$ Kinesiology, Katholieke Universiteit Leuven, \\ Belgium.
}

\begin{abstract}
Little is known about the ability of blind people to cross obstacles after they have explored haptically their size and position. Long-term absence of vision may affect spatial cognition in the blind while their extensive experience with the use of haptic information for guidance may lead to compensation strategies. Seven blind and 7 sighted participants (with vision available and blindfolded) walked along a flat pathway and crossed an obstacle after a haptic exploration. Blind and blindfolded subjects used different strategies to cross the obstacle. After the first 20 trials the blindfolded subjects reduced the distance between the foot and the obstacle at the toe-off instant, while the blind behaved as the subjects with full vision. Blind and blindfolded participants showed larger foot clearance than participants with vision. At foot landing the hip was more behind the foot in the blindfolded condition, while there were no differences between the blind and the vision conditions. For several parameters of the obstacle crossing task, blind people were more similar to subjects with full vision indicating that the blind subjects were able to compensate for the lack of vision.
\end{abstract}

Keywords: blind, gait, obstacle crossing, toe clearance, visual information

$\mathbf{T}$ here is evidence that blind people learn to adjust their gait to be as efficient as possible given the absence of vision. For example in late blind, the gait pattern gradually changes so as to approximate the gait patterns of congenitally blind people as the duration of visual loss progresses (Nakamura, 1997). Vision is important for overground locomotion and crucial in situations such as negotiating stairs or obstacles, and in its absence, people adopt several cautious strategies. For situations with reduced or disrupted vision, such strategies include slower speeds and increased foot clearance (Elliott, 2014). In addition to these, it has been reported that there is an increased ankle plantar flexion angle range during the loading response, indicative of increased use of tactile feedback from the plantar aspect of the foot (Hallemans, Ortibus, Meire, \& Aerts, 2010). Such strategies are also seen when subjects walk down or up a set of stairs without having normal visual feedback. In such cases one can observe that subjects with visual impairments tend to keep their COM (center of mass) longer over the BOS (base of support; Heasley, Buckley, Scally, Twigg, \& Elliott, 2004), which poses possible instability (see Elliott, 2014).

The experiments mentioned so far were performed during normal walking. When faced with obstacles, normal sighted subjects with lower visual field restriction or with no vision slow down their gait to be able to use haptic information (provided by a cane) to allow for greater time for haptic exploration (Patla, Davies, \& Niechwiej, 2004). In contrast, little is known about adaptations for complex walking conditions, such as when avoiding obstacles, in blind people. The question as to how fully blind or blindfolded people face the problem of avoiding obstacles when walking has received limited attention, for a recent review see (Kolarik, Cirstea, Pardhan, \& Moore, 2014). Some work reported that blindfolded sighted adults could detect large scale obstacles (Masonite boards) set up perpendicularly to their line of travel when they approached it up to about $2 \mathrm{~m}(6 \mathrm{ft}$; Ammons, Worchel, \& Dallenbach, 1953). According to these authors the presence of auditory information (echolocation) was at the basis of this obstacle sense. For smaller obstacles (e.g., boxes) such auditory feedback is reduced, yet blind children were found to spend significantly more time in the region before the obstacle than in the region after it, indicating they still had some ability to detect these obstacles (Ashmead, Hill, \& Talor, 1989). These authors claimed that their data can be explained by sound information provided by their own footsteps. One would expect that blind people are especially proficient in this skill. Indeed, in adults, Strelow and Brabyn (1982) showed that the blind have a greater skill than blindfolded sighted subjects in using auditory cues for spatial guidance. It is conceivable that the same applies for haptic cues but this has not been investigated yet. The blind may be deficient in spatial cognition because this relies heavily on vision. On the other hand, it might be possible that the blind are better in using haptic cues for safe navigation. In addition, the possibility exists that blind people learn to avoid obstacles by using alternative safe strategies. For example, in studies on subjects with impaired vision it is common to see some adjustments when crossing obstacles, such as higher toe-clearance and increased distance between foot and obstacle

Correspondence address: Arturo Forner-Cordero, Biomechatronics Lab, Mechatronics Department, Escola Politécnica da Universidade de São Paulo, Av. Prof. Mello Moraes, 2231 Cidade Universitaria 05508-030 - São Paulo-SP - Brasil. e-mail: aforner@usp.br 
at foot placement (Rhea \& Rietdyk, 2007; Timmis \& Buckley, 2012). The same effect was reported if the visual information was removed when approaching the obstacle (Mohagheghi, Moraes, \& Patla, 2004) or when visual information was replaced by haptic information (Patla et al., 2004).

In view of the expected adaptation to obstacle avoidance in the blind, it would be of interest to compare the behavior in blind persons and in people with a shortterm vision loss (e.g., blindfolded). When given the same haptic information about the obstacle, the question arises whether people that have been blind for several years perform better at avoiding obstacles after they had a chance to explore them haptically. To examine this question, a comparison was made between sighted people (with or without blindfolding) and blind subjects. Blind and blindfolded groups were allowed to first obtain haptic information of the obstacle and then were brought back some steps before trying to cross the obstacle. This requires the subjects to store an internal representation of the obstacle in relation to their body. In studies on cats it was shown that this ability exists (McVea \& Pearson, 2006, 2007) and that it depends on parietal cortex (McVea, Taylor, \& Pearson, 2009; Marigold \& Drew, 2011). Humans have the capability to store this information for more than $2 \mathrm{~min}$ (Lajoie, Bloomfield, Nelson, Suh, \& Marigold, 2012). In one of these studies (Heijnen, Romine, Stumpf, \& Rietdyk, 2014) the effects of position memory was investigated by replacing an obstacle by high-contrast tape after subjects had avoided the obstacle 25 times. No changes in foot placement were observed, suggesting that the position cue provided salient online information to guide foot placement. This finding agrees with previous research that reported that humans control the obstacle crossing task guiding the leading limb on a feedforward manner using visual information (Moraes, Lewis, \& Patla, 2004; Santos, Moraes, \& Patla, 2010).

The aim of the study was to test whether blind persons perform better and/or differently from blindfolded subjects at crossing remembered obstacles when relying on haptic information to store information about obstacle features in relation to the body. It was hypothesized that blind subjects would integrate haptic information to cross obstacles with lower error rates than blindfolded persons and with more similar and appropriate obstacle crossing patterns to participants with vision than blindfolded subjects because of longer experience with the use of haptic information without vision. To test this, a set of experiments was performed using obstacle crossing with blind and blindfolded participants. Control sighted subjects with full vision were tested as well to obtain baseline data (the same subjects were used for the sighted and blindfolding conditions). This allowed us to determine the "optimal conditions" with which all other conditions could be compared.

\section{Materials and Methods}

\section{Participants}

Seven blind participants and seven sighted participants volunteered in this study. Blind participants had all longterm (several years), acquired blindness and were classified as type B1 according to the International Blind Sport Federation criteria. B1 class includes individuals in the range from no light perception in either eye to light perception, but inability to recognize the shape of a hand at any distance or direction (Winnick, 2005). Sighted and blind participants were age and height-matched. They had, respectively, mean age of 39.3 years $(S D=11.5$ years) and 39.3 years $(S D=11.7$ years $)$ years and mean height of $173.8(S D=4.9)$ and $172.1(S D=8.0) \mathrm{cm}$. All participants provided informed consent and the testing was conducted according to the ethical guidelines of São Paulo State University.

\section{Apparatus}

A digital video camera (Sony DCR DVD 205, $60 \mathrm{~Hz}, 1$ Megapixel; Sony Corp., San Diego, CA) recorded the participants performing the task. A software package (Ariel Performance Analysis System; Ariel Dynamics Inc., Trabuco Canyon, CA) was used for a two-dimensional kinematical analysis. The camera was placed perpendicularly to the sagittal plane of the subject at $3 \mathrm{~m}$. The vision field comprised at least one step before the obstacle and one step behind it, less than $3 \mathrm{~m}$ in total, providing a resolution lower than $0.26 \mathrm{~cm}$. Matlab (Version 7.10.0.499 [R2010a]; The MathWorks Inc., (SPSS: Release 17.0.1, SPSS, Inc.) Natick, MA) and SPSS Inc. were used respectively for processing the motion data, parameter extraction with custom-made programs and statistical analysis.

\section{Procedure and Task}

All participants walked barefoot along a $5 \mathrm{~m}$ flat pathway with an obstacle of $26 \mathrm{~cm}$ height located at $2 \mathrm{~m}$ (about four to five steps) from the starting point. The obstacle height represented between $30.4 \%$ and $32.9 \%$ of the leg length of the subjects. The obstacle consisted of a low weight bar ( $400 \mathrm{~g}$, length of $88 \mathrm{~cm}$, diameter of $6 \mathrm{~cm})$ supported on both sides in such a way that a light touch could remove it, thus guaranteeing the safety of the volunteers. The light bar did not provide any auditory cue because it barely reflects acoustic signals, as it was shown that auditory obstacle sense deteriorates markedly when smaller targets are used (Strelow \& Brabyn, 1982).

If the obstacle was changed or knocked off during the exploration or during a trial (e.g., when tripping) it was replaced carefully (and silently) by the experimenter. The sighted participants completed 30 successful obstacle crossings with their right leg as leading limb, first blindfolded 
and then with vision. Similarly, the seven blind participants also performed the same task thirty times. If a trial was not successful, it was recorded as an error and another trial was measured in order to have a total of 30 successful crossings that were used to compare performance between the three groups. Gait speed was self-selected. Blind and blindfolded participants were allowed to explore the experimental environment, walking and touching the obstacle (either with their feet or their hands or both), prior to starting and between trials. The use of a cane was not allowed. Care was taken that subjects had approximately the same time to explore the obstacle and, although they had no specific time limit, they spent less than $10 \mathrm{~s}$. After exploring the obstacle without vision, they were guided to go to the starting point and walk to cross the obstacle, stopping two strides after crossing. They were guided to the starting point again to be ready for the next trial. Subjects were positioned such that it would take them about four or five steps to reach the obstacle but they were instructed not to count the steps themselves.

\section{Data Analysis and Dependent Variables}

Figure 1 shows a line drawing representing a two-dimensional model of the swing leg during the obstacle crossing task with anteroposterior and vertical hip-foot distances, toe clearance and the obstacle identified.

The motion of the leading limb was video recorded at $60 \mathrm{~Hz}$. Five markers were placed on the right limb at the hip (greater trochanter), knee (lateral epicondyle), ankle (lateral malleollus), rearfoot (calcaneus tuberosity), and forefoot (fifth metatarsal joint). The analysis included the leading limb's step over the obstacle which had its onset at the instant the crossing foot left the ground (takeoff) and its offset at the instant this foot touched the ground again (landing). The motion data were filtered with a fourth order recursive Butterworth filter with a cut-off frequency of $8 \mathrm{~Hz}$. A crossing error or "failure" was defined as a contact with the obstacle. It must be noted that no loss of balance occurred after touching the obstacle. Only the motion data of successful trials were analyzed.

The following dependent variables were obtained from the motion data and defined as shown in Figure 1:

- Time of Forefoot Clearance (TFC). Absolute time duration in seconds between toe-off and the instant when the forefoot crosses the obstacle (measured when the forefoot marker was aligned with the obstacle marker in the anteroposterior axis).

- Time of Heel Clearance (THC). Absolute time duration in seconds between toe-off and the instant when the heel crosses the obstacle (measured when the heel marker was aligned with the obstacle marker in the anteroposterior axis).

- TFC percentage. The instant when the forefoot marker crosses the obstacle given as a percentage of the total duration between toe-off and foot landing.

- THC percentage. The instant when the heel marker crosses the obstacle given as a percentage of the total duration between toe-off and foot landing.

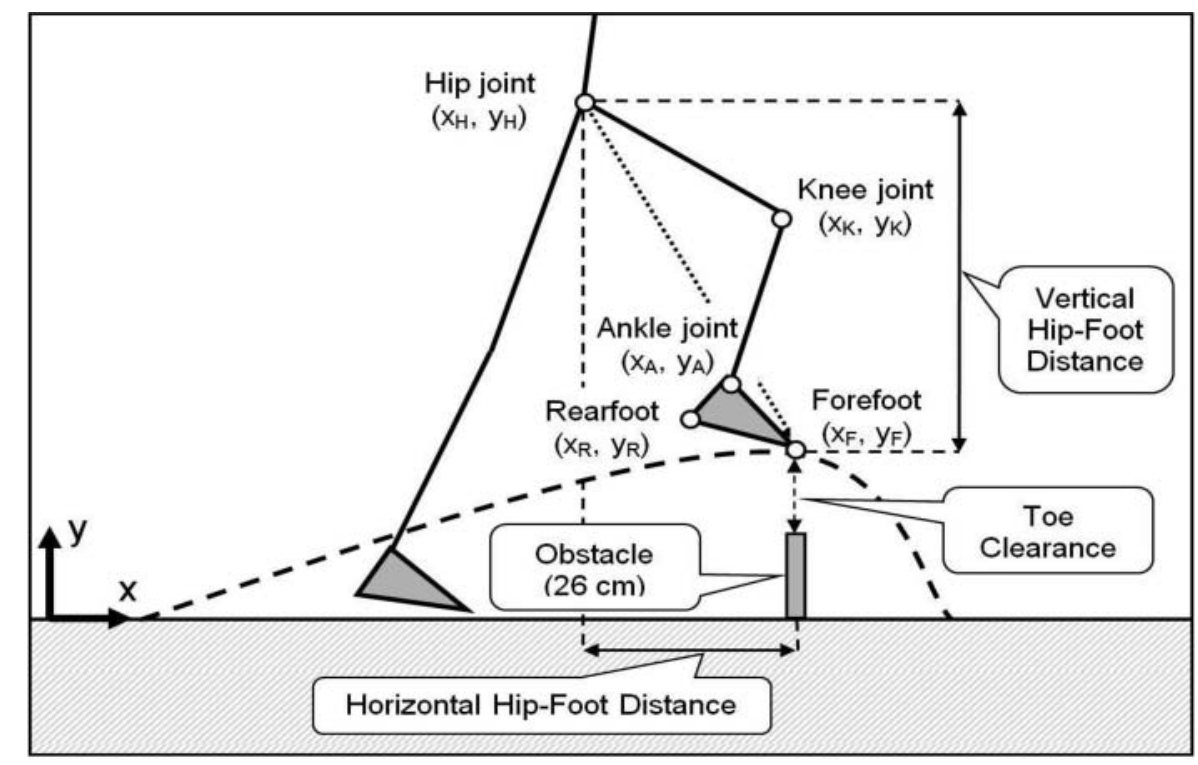

FIGURE 1. Line drawing that represents the swing leg and defines the coordinate system used during obstacle crossing and the corresponding dependent variables. 
- Forefoot Clearance. Vertical height from the forefoot marker to the obstacle measured at the TFC (given in centimeters, as shown in Figure 1).

- Heel clearance: Vertical height from the heel marker to the obstacle that occurs at the THC.

- Lead Swing Velocity: Average velocity of the leading limb step over the obstacle calculated by dividing step length by step duration.

The second group of variables analyzed was related to the "strategy" used to cross the obstacle (hip and foot positions at different instants during the step). In particular, it was of interest to investigate the hip holding back strategy as described by Patla and Rietdyk (1993). In this strategy the hip is kept behind while aiming forward with the foot in order to cross obstacles of increasing height. In this way the center of mass is closer to the supporting limb and weight transfer of weight is delayed.

The hip-foot distance described the effective leg length in vertical and anteroposterior axis. These variables were normalized to subject height:

- Normalized heights of hip and foot. Vertical position (Y) of the hip and forefoot markers divided by subject height at TFC.

- Normalized hip foot distance. Distance between the hip and the leading forefoot markers in the anteroposterior (NXHF) and vertical (NYHF) axes divided by subject height. These distances were calculated at three different instants: toe-off, TFC, and foot landing, according to the following equations:

$\mathrm{NXHF}=(\mathrm{xforefoot}-\mathrm{xhip}) /$ height $($ this distance

is positive when the hip is behind the foot)

$\mathrm{NYHF}=($ yhip - yforefoot $) /$ height

where xforefoot and xhip are the anteroposterior positions of the forefoot and hip markers, respectively and yforefoot and yhip are the vertical positions of the forefoot and hip markers, respectively.

- Normalized anteroposterior foot obstacle distance at toe-off. Distance between the forefoot of the leading leg and the obstacle at the toe-off instant of the crossing step.

$$
\mathrm{NHFO}=(\text { xobstacle }- \text { xforefoot }) / \text { height }
$$

As the heel of the lead foot is typically closer to the obstacle than the toe during lead obstacle crossing, we also calculated the minimum foot clearance (which was either the toe or the heel clearance, whichever was smallest;
Chen, Ashton-Miller, Alexander, \& Schultz, 1991; Heijnen, Muir, \& Rietdyk, 2012; Loverro, Mueske, \& Hamel, 2013).

- Normalized anteroposterior heel distance to obstacle at foot landing: Distance between the heel of the leading forefoot and the obstacle at foot landing.

\section{Statistical Analyses}

The difference in the number of errors while crossing the obstacle for blind and blindfolded was analyzed by means of chi-square test. To test the effect of availability of visual feedback, several descriptive statistical parameters from the step and strategy variables were obtained, including means, standard deviations, and medians. Kolmogorov-Smirnov tests were used to evaluate normality and most of the distributions were not normal. As the variances were also not homogeneous, it was chosen to perform nonparametric tests (Mann-Whitney $U$ test) between pairs of groups: blind and blindfolded, vision, and blind. For the comparison between blindfolded and vision a nonparametric test for within group comparison (the Wilcoxon signed rank test), was used as it corresponds to comparing the same group of subjects under different conditions (first blindfolded and afterwards with vision).

Finally, a separate analysis was made in order to assess the behavior of the foot-obstacle distance as a function of the sequence of thirty trials. These thirty trials were grouped in three nonoverlapping blocks of ten trials and a nonparametric pairwise comparison was made for each one of the three blocks between groups. A nonparametric test (Mann-Whitney $U$ test) was used to compare blind and blindfolded and also vision and blind for each block, hence for the first block of 10 trials (from 1 to 10), then for the second (trials 11-20) and, finally for the third block (trials 21-30). A level of $p<.05$ was chosen for significance in all the tests.

\section{Results}

Normal sighted subjects made no errors. In contrast, blindfolded subjects touched the obstacle in $17.7 \%$ of trials, as compared to $12.5 \%$ for the blind subjects. In the blind group there was one subject with zero errors, while in the blindfolded group none of the subjects achieved this level of performance. Nevertheless, the difference between blind and blindfolded was not significant according to the chi-square tests.

The failures occurred more frequently by contacts with the trailing limb as reported in previous literature (Heijnen et al., 2012). Nevertheless, the percentages of errors with the leading limb were substantial. For the blind population $39.2 \%$ of the errors occurred with the leading limb, while for the blindfolded participants leading limb error reached $35.1 \%$. 
TABLE 1. $U, Z$, and $p$ values of Significant Comparisons (Used to Define Significant Signs in Table 1)

\begin{tabular}{|c|c|c|c|c|c|c|c|c|c|}
\hline \multirow[b]{3}{*}{ Dependent variables } & \multicolumn{9}{|c|}{ Comparisons } \\
\hline & \multicolumn{3}{|c|}{ Blind $\times$ Blindfolded (a) } & \multicolumn{3}{|c|}{ Blind $\times$ Vision $(b)$} & \multicolumn{3}{|c|}{ Blindfolded $\times$ Vision $(\mathrm{c})$} \\
\hline & $U$ & $Z$ & $p$ & $U$ & $Z$ & $p$ & $U$ & $Z$ & $p$ \\
\hline $\begin{array}{l}\text { Toe clearance time } \\
(\mathrm{s})^{\mathrm{a}+, \mathrm{b}^{*}, \mathrm{c}^{*}}\end{array}$ & 18336 & -2.766 & 0.006 & 12565 & -7.519 & $<0.001$ & 15315 & -5.357 & $<0.001$ \\
\hline $\begin{array}{l}\text { Heel clearance time } \\
(\mathrm{s})^{\mathrm{b}^{*}, \mathrm{c}^{*}}\end{array}$ & & & ns & 13429 & -6.817 & $<0.001$ & 15348 & -5.329 & $<0.001$ \\
\hline $\begin{array}{l}\text { Toe clearance time percentage } \\
(\%)^{b^{* *}, c^{*}}\end{array}$ & & & ns & 8083 & -11.140 & $<0.001$ & 4768 & -13.859 & $<0.001$ \\
\hline $\begin{array}{l}\text { Heel clearance time percentage } \\
(\%)^{b^{*}, c^{*}}\end{array}$ & & & ns & 6589 & -12.349 & $<0.001$ & 4823 & -13.815 & $<0.001$ \\
\hline $\begin{array}{l}\text { Toe clearance } \\
(\mathrm{cm})^{b^{*}, c^{*}}\end{array}$ & & & ns & 12441 & -7.611 & $<0.001$ & 8601 & -10.766 & $<0.001$ \\
\hline $\begin{array}{l}\text { Heel clearance } \\
\qquad(\mathrm{cm})^{a^{*}, b^{*}, c^{*}}\end{array}$ & 17652 & -3.319 & 0.001 & 10680 & -9.037 & $<0.001$ & 6493 & -12.467 & $<0.001$ \\
\hline $\begin{array}{l}\text { Lead swing velocity } \\
\qquad(\mathrm{cm} / \mathrm{s})^{\mathrm{b}^{*}, \mathrm{c}^{*}}\end{array}$ & & & ns & 11821 & -8.113 & $<0.001$ & 11986 & -8.035 & $<0.001$ \\
\hline $\begin{array}{l}\text { Norm. hip height at toe clearance } \\
\text { time }^{a^{*}, c^{*}}\end{array}$ & 16702 & -4.901 & $<0.001$ & & & ns & 12183 & -7.877 & $<0.001$ \\
\hline $\begin{array}{l}\text { Norm. hip height at heel clearance } \\
\text { time }^{a^{*}, c^{*}}\end{array}$ & 17041 & -3.815 & $<0.001$ & & & ns & 10383 & -9.329 & $<0.001$ \\
\hline $\begin{array}{l}\text { Norm. foot height at toe clearance } \\
\text { time }^{b^{*}, c^{*}}\end{array}$ & & & ns & 13792 & -6.517 & $<0.001$ & 9394 & -10.127 & $<0.001$ \\
\hline $\begin{array}{l}\text { Norm. heel height at heel clearance } \\
\text { time }^{a+, b^{*}, c^{*}}\end{array}$ & 18493 & -2.635 & 0.008 & 11540 & -8.340 & $<0.001$ & 8035 & -11.223 & $<0.001$ \\
\hline $\begin{array}{l}\text { Norm. anteroposterior hip-foot } \\
\text { distance at toe-off }\end{array}$ & & & ns & & & ns & & & ns \\
\hline $\begin{array}{l}\text { Norm. anteroposterior hip-foot } \\
\quad \text { distance at toe clearance time } \\
{ }^{a+}, c^{*}\end{array}$ & 18655 & -2.504 & 0.012 & & & ns & 12625 & -7.520 & $<0.001$ \\
\hline $\begin{array}{l}\text { Norm. anteroposterior hip-foot distance } \\
\text { at foot landing } \mathrm{a}^{*}, \mathrm{c}^{*}\end{array}$ & 13360 & -6.807 & $<0.001$ & & & ns & 9537 & -10.012 & $<0.001$ \\
\hline $\begin{array}{l}\text { Norm. vert. hip-foot } \\
\text { distance at toe-off } \mathrm{a}^{*}, \mathrm{~b}^{*}, \mathrm{c}^{*}\end{array}$ & 9582 & -9.877 & $<0.001$ & 11345 & -8.498 & $<0.001$ & 17.547 & -3.548 & $<0.001$ \\
\hline $\begin{array}{l}\text { Norm. vert. hip-foot distance at toe } \\
\text { clearance time }{ }^{a+,} b^{*}, c^{*}\end{array}$ & 18462 & -2.661 & 0.008 & 10253 & -9.382 & $<0.001$ & 13401 & -6.894 & $<0.001$ \\
\hline $\begin{array}{l}\text { Norm. vert. hip-foot distance at } \\
\text { foot landing }{ }^{\mathrm{a}^{*}, \mathrm{~b}^{*}, \mathrm{c}^{*}}\end{array}$ & 12438 & -7.556 & $<0.001$ & 13542 & -6.719 & $<0.001$ & 17161 & -3.860 & $<0.001$ \\
\hline $\begin{array}{l}\text { Norm. anteroposterior forefoot obstacle } \\
\text { distance at toe-off } \mathrm{f}^{\mathrm{a}, \mathrm{c}^{*}}\end{array}$ & 18451 & -2.670 & 0.008 & & & ns & 16126 & -4.695 & $<0.001$ \\
\hline $\begin{array}{l}\text { Norm. anteroposterior heel obstacle } \\
\text { distance at foot landing } \mathrm{a}^{\mathrm{a}+, \mathrm{b}^{*}, \mathrm{c}^{*}}\end{array}$ & 18869 & -2330 & 0.020 & 16538 & -4.293 & $<0.001$ & 12469 & -7.646 & $<0.001$ \\
\hline
\end{tabular}

Note. Significant differences between blind and blindfolded (a), blind and vision (b), and blindfolded and vision (c) at .02 (+) or .001 (").

Step parameters were analyzed as well. Table 1 summarizes the descriptive statistics for these variables. Note that the differences between blindfolded and vision groups were significant for all the variables, as expected.

With vision, the forefoot and heel clearance were significantly smaller (Table 1); TFC also decreased significantly (Figure 2A) and the swing velocity was faster (Figure 2B). The larger TFC indicated that it took longer for the blind longer to reach the obstacle after toe-off than the blindfolded. Also the blindfolded took significantly longer with respect to vision. The difference between blind and blindfolded $(U=18336, Z=-2.766, p=.006)$ was larger than the difference between blindfolded and vision (see Figure 2A and Table 1).

Forefoot and heel clearance were larger for the blindfolded than for the blind but the difference was only significant for the heel clearance $(U=17652, Z=-3.319, p<$ $.001)$. Forefoot and heel clearance are a function of flexion of the leg but it may also depend on hip position. To evaluate the latter, the hip and forefoot height were compared (Figure 3).

Both blind and blindfolded had the highest forefoot height at the moment of forefoot clearance (Figure 3), yet only the blindfolded had also increased hip height 

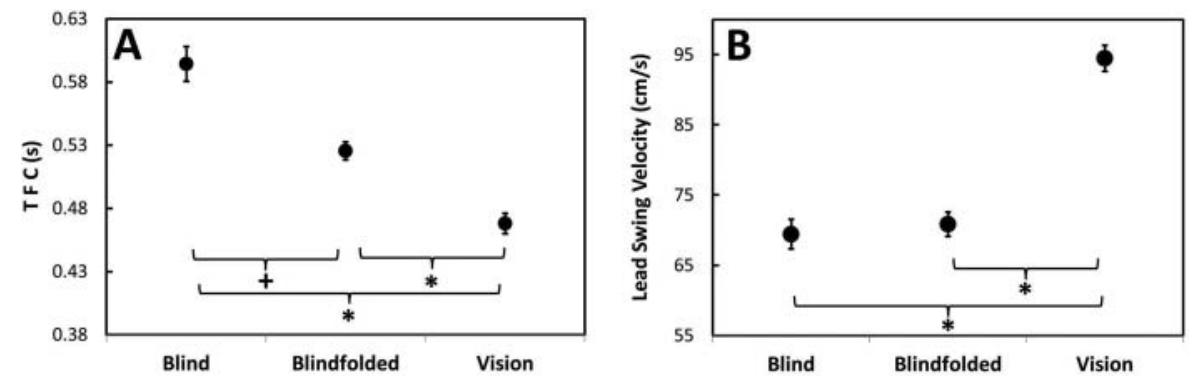

FIGURE 2. Mean \pm standard error of time of forefoot clearance-TFC (A) and lead swing velocity (B) of blind, blindfolded, and vision groups/conditions. Significant differences are indicated by asterisk $(p<.001)$ and plus sign $(p<.02)$.

(Figure 4; $U=16702, Z=-4.091, p<.001$ ). In contrast the blind combined a high foot with a low hip height.

\section{Strategies}

Differences between blind and blindfolded were apparent when analyzing the strategies used to cross the obstacle successfully (Figure 5). At foot landing, which occurred with the forefoot (thus explaining the choice of the forefoot marker to calculate the anteroposterior foot distance), the hip was significantly more behind the foot in the blindfolded condition than in the blind $(p<.001)$, while there were no differences between the blind and the vision conditions (see Figure 5B).

\section{Sequence Dependent Changes}

There were other differences between blind and blindfolded but these differences depended on trial sequence. One important factor in obstacle crossing is the starting position before crossing (forefoot obstacle distance at toeoff). If this distance is too short then the risk to fail is increased (Heijnen et al., 2012). When evaluating this parameter across trials it was striking that only the blindfolded seemed to take a higher risk by nearing the obstacle closely after the first 16 trials while in the previous 16 trials both blind and blindfolded had small forefootobstacle distances (Figure 6A).

To verify the idea that the errors in the blindfolded were linked to the smaller distance of the foot to the obstacle the data were inspected for occurrence of failures. There was no obvious increase in error rate in the blindfolded subjects during the period when they approached the obstacle (trials 17-30).

The distance between heel and obstacle at foot landing after crossing the obstacle was plotted as well (Figure 6B). In the absence of vision this distance increased after repetitions, while for the vision condition it was relatively constant (Figure 6B). In the blindfolded, the increase in this distance at landing (Figure 6B) occurred around trial 20, hence at about the same time as when they decreased the distance at toe-off (Figure 6A).

The blindfolded came closest to the obstacle (Figure 7A). The nonparametric tests showed that there were significant differences between the blindfolded and blind groups $(U=18451, Z=-2.670, p=.008)$ and also between the blindfolded and vision $(U=16126$, $Z=-4.695, p<.0001)$. Note that there were no differences between the blind and vision groups. For the heel
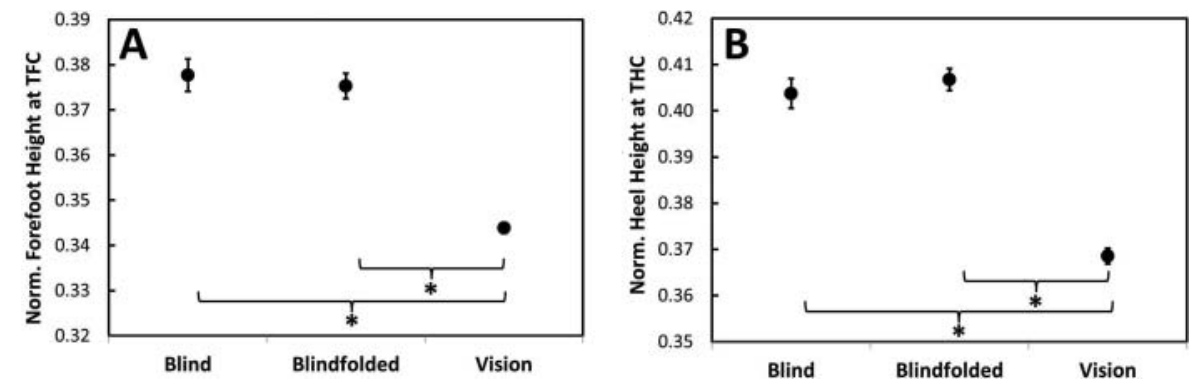

FIGURE 3. Mean \pm standard error of normalized forefoot height at TFC (A) and normalized heel height at time of heel clearance-THC (B) of blind, blindfolded, and vision groups/conditions. Significant differences are indicated by asterisk $(p<.001)$. 


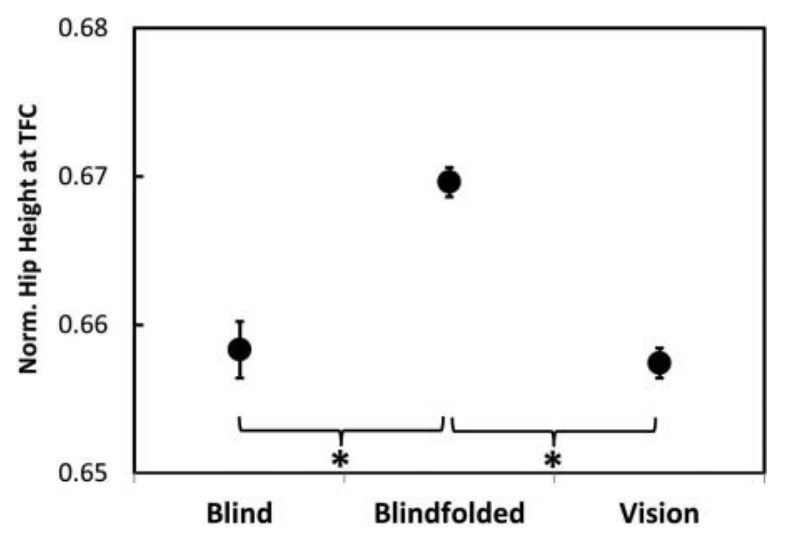

FIGURE 4. Mean \pm standard error of normalized hip height at time of forefoot clearance-TFC of blind, blindfolded, and vision groups/conditions. Significant differences are indicated by asterisk $(p<.001)$.

obstacle distance at landing after crossing the obstacle (Figure 7B) the analyses revealed differences between the three groups/conditions. The vision group showed a smaller distance to the obstacle than the blind and the blindfolded. Also, the blind had a significantly smaller distance than the blindfolded (Figure 7B).

Regarding the analysis of the normalized forefootobstacle distance at toe-off, using nonoverlapping blocks of ten trials, the blindfolded showed significantly smaller distances than the blind only in the final block of ten trials $(U=1709, Z=-2.859, p=.004)$. In contrast, in this block there were no significant differences between blind and vision. As for the comparison between vision and blindfolded, there were significantly larger distances in the vision group than in the blindfolded in all the three blocks of trials.

These differences in "foot obstacle distance at toeoff" were not related to differences in speed (not illustrated). All groups/conditions increased their speed over the first 10 trials but they remained at a stable speed afterward. The vision group was always faster. After the first 17 trials there was no speed difference between blind and blindfolded.

\section{Discussion}

This study investigated obstacle avoidance after haptic exploration in blind and blindfolded volunteers, and it was found that most trials could be completed successfully when vision was absent. This finding supports the hypothesis that haptic information was sufficient, thereby confirming the results of Patla et al. (2004). The data also show that blind people cross obstacles differently from blindfolded volunteers, supporting the hypothesis that long-term lack of vision results in an improved management of haptic information. When failures occurred they most frequently involved the trail limb (in line with Heijnen et al. [2014]).

The error rates found here were very similar to those of Patla (1998) and lower than in other experiments (Patla and Greig [2006], noting error rates of about 50\%), because in the present study the participants were allowed to explore the experimental environment, walking and touching the obstacle prior to starting and in between trials. This underscores that haptic information can be used to improve performance. Such haptic information is even more important for the blind participants, since they performed the task with lower number of errors, indicating a more effective use of haptic information.

A new major result of the present study is that the blindfolded subjects differed from blind ones in crossing remembered obstacles, following haptic exploration. This fact underscores the presence of an adaptation strategy in longterm blind people. The present data focused on the successful trials and showed that there were differences in the method used by the leading limb to avoid obstacles.

In general, as compared to sighted controls, both blind and blindfolded participants used a higher forefoot clearance to safely cross the obstacle but these two groups differed in other aspects. The significantly larger TFC found in the blind group with respect to the blindfolded can be
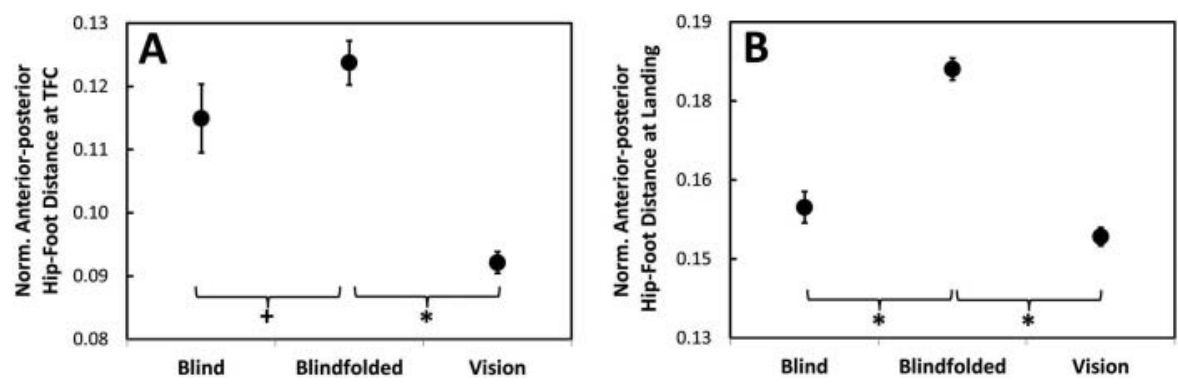

FIGURE 5. Mean of normalized anterior-posterior hip-foot distance at time of forefoot clearance-TFC (A) and normalized anterior-posterior hip-foot distance at landing (B) of blind, blindfolded, and vision groups/conditions. Significant differences are indicated by asterisk $(p<.001)$ and plus sign $(p<.02)$. 

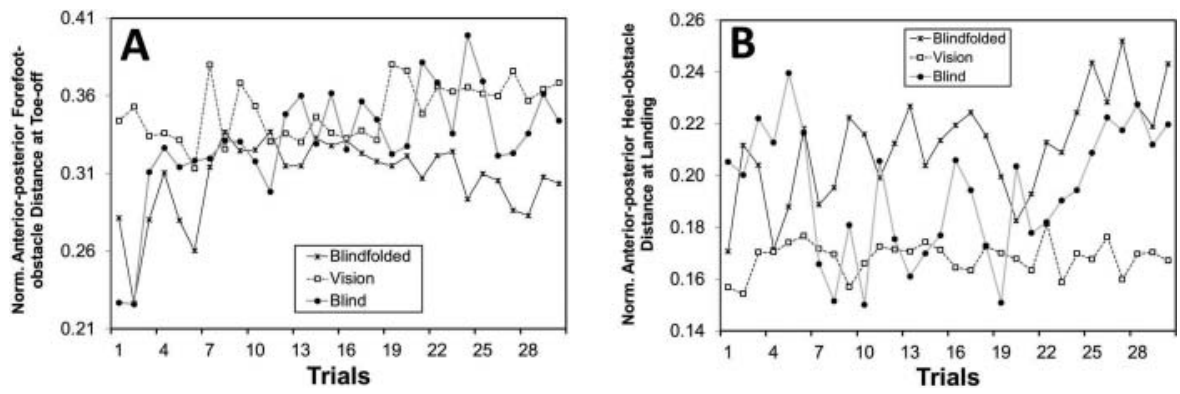

FIGURE 6. Mean of normalized anterior-posterior forefoot-obstacle distance at toe-off (A) and normalized anterior-posterior heelobstacle distance at landing (B) over 30 trials of blind, blindfolded, and vision groups/conditions.

explained by a different strategy to negotiate the obstacle, which is crossed later during the swing phase. This could be related to a preference for a lowering strategy, that appears in mid- or late swing perturbations, in case the foot contacts the obstacle. At the time of forefoot clearance (TFC) the hip height in the blind was similar to that of the vision group but lower than in the blindfolded. This appears to indicate a more energetically efficient strategy to cross the obstacle by the blind as compared to the blindfolded (Begg, Best, Dell'Oro, \& Taylor, 2007; Lu, Chen, \& Chiu, 2012; Rodrigues, Forner-Cordero, Garcia, Zago, \& Ferasoli, 2009). The increase in hip height is reminiscent of hip hiking as is observed when high obstacles need to be crossed (Patla \& Prentice, 1995) or in unperturbed gait in a number of pathologies (patients with stroke or with knee replacement; see Byrne \& Prentice, 2003). Nevertheless, the assessment of hip hiking would require bilateral measurements, which are left for further research. The blind were also more similar to the vision group with respect to another relevant parameter, the anteroposterior distance between foot and obstacle at the time of take-off. Both groups differed from the blindfolded. This difference was speed-independent and did not appear from the first trial onwards but became apparent at about half-way the sessions. The blindfolded group then progressively approached the obstacle more closely, thereby increasing the risk of touching the obstacle during crossing. Indeed, in previous studies it was seen that touching the obstacle was observed when subjects came too close to the obstacle for the starting point of the crossing step either in no vision conditions or with a visual sampling of the scene (Chou \& Draganich, 1998; Patla \& Greig, 2006). In this respect, it must be noted that when the lead limb is placed too far away from the obstacle, there is also an increased risk of contact with the heel of the lead foot (Galna, Murphy, \& Morris, 2010; Muir, Haddad, Heijnen, \& Rietdyk, 2015). The analysis of the heel-obstacle distance at foot contact after crossing the obstacle revealed that in the absence of vision, this distance is larger than in the vision group. This mechanism could be related to the larger forefoot obstacle clearance found in the blind and blindfolded groups and could be explained as a safety measure, induced by the uncertainty about the obstacle position and height.

Moreover, the forefoot obstacle distance shows another surprising behavior when analyzed in blocks of consecutive trials. While blind participants in the last ten trials converged to similar values as the vision group, the blindfolded clearly diverged to smaller forefoot obstacle distances. It is
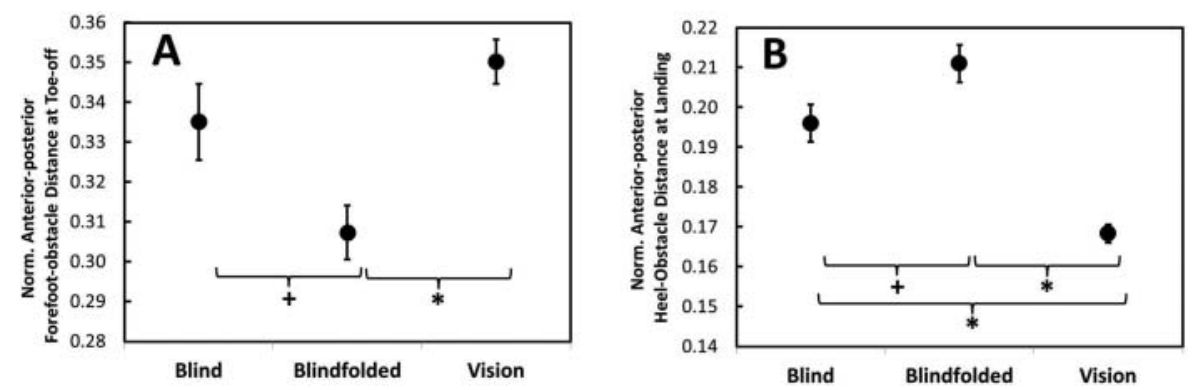

FIGURE 7. Mean \pm standard error of normalized anterior-posterior forefoot-obstacle distance at toe-off (A) and normalized anterior-posterior heel-obstacle distance at landing $(\mathbf{B})$ of blind, blindfolded, and vision groups/conditions. Significant differences are indicated by asterisk $(p<.001)$ and plus sign $(p<.02)$. 
possible that they were trying to avoid contacting the obstacle with the trailing limb, which would reflect a more conservative strategy of the blindfolded as compared to the blind.

It is of interest to note that the anteroposterior forefoot distance to the obstacle at toe-off for the blindfolded subjects showed a small decrease across trials, differently from that of the blind and vision groups (Figure 7). This is reminiscent of the data on another parameter, namely minimal foot clearance (observed on a treadmill with repeated obstacles [Erni \& Dietz, 2001] or overground [Heijnen et al., 2014]). In these studies one can also see a gradual shift to more "risky" behavior as trials are repeated. Furthermore, in the study of Heijnen et al. (2012) it was shown that a main cause of errors (e.g., spontaneous contacts with a fixed, visible obstacle) was a progressive decrease in foot clearance until an error occurred. For future experiments it would be of interest to see whether a gradual decrease in foot obstacle distance could lead to a similar increase in failure rate in the blindfolded. This is likely as the present data already showed that blindfolded subjects made more errors than the blind subjects, consistent with a closer approach to the obstacle.

Another aspect of obstacle crossing, in which blind and blindfolded differed, concerned the position of the foot with respect to the hip at the instant of foot landing, which occurred mainly with the forefoot, after crossing the obstacle. In this variable, there were no differences between blind and vision participants, while there were significant differences with the blindfolded (see Figure 5b). The latter group kept the hip behind while aiming forward with the foot. Patla and Rietdyk (1993) observed this same strategy when normal sighted subjects were asked to cross obstacles of increasing height. They mentioned that this can be seen as holding the hip closer to the stance limb as the higher obstacles needed to be crossed; hence it is a method to bring the center of mass closer to the supporting limb. Transfer of weight is delayed to ensure that the ipsilateral limb has safely crossed the obstacle. This strategy likely represents a compromise between cautiousness and gait efficiency during the crossing, as reported by other authors (Begg et al., 2007; Lu et al., 2012). The anteroposterior distance between hip and the feet determines the point of application of the ground reaction forces, thus the moment arm to apply trunk torques when the foot is placed on the ground (Forner Cordero, Koopman, \& van der Helm, 2004, 2014). In case the foot impacts the obstacle and a trip occurs, it is advantageous to keep the foot more ahead of the hip to perform a lowering strategy because a larger moment arm allows larger trunk torques and there is more time to transfer the load to the limb. It can also be interpreted that the foot positions determine the base of support $(\mathrm{BoS})$ and the margin of stability can be defined as the minimum distance from the anteroposterior position of the center of mass to the boundaries of the BoS (Hof, Gazendam, \& Sinke, 2005). Therefore, keeping the foot ahead of the hip facilitates the execution of a lowering recovery strategy, one of two strategies seen in tripping responses (Eng, Winter, \& Patla, 1994; Forner-Cordero et al., 2004, 2014; Schillings, Van Wezel, Mulder, \& Duysens, 1999). Hence this would argue for a more cautious approach taken by the blindfolded (Figure 5b). This may come at a cost though since the support of the trailing limb could be prolonged, thus leading to potential instability. It is suggested that a measure of how confidently a subject approaches an obstacle could be given by the time spent to reach the obstacle. In a study of the effects of stepping down a set of stair in subjects with blurred vision it was found that a cautious strategy consisted in holding back the body longer when stepping down (Heasley et al., 2004). This included longer single-limb support by the trailing limb, which was considered to represent a danger for stability, particularly in the mediolateral direction. It is interesting to note that keeping the hip backwards while crossing the obstacle, which is the behavior of the blindfolded group, resembled the control strategy of bipedal robots using the Zero Moment Point stability criterion (Rossi, Rodrigues, \& Forner-Cordero, 2014).

The heel clearance and the time of heel clearance were used to assess the possibility of the foot contacting the obstacle with the heel. However, this was not really an issue because it was consistently found that the heel clearance was larger than the forefoot clearance.

While the difference in the mean number of errors was modest (and below significance level) it is nevertheless surprising that blind people performed so well in the task. A possible reason could be provided by the differences in crossing behavior outlined previously.

First, it may be that blind people are better in the nonvisual detection of obstacles. It is known that blind people can successfully use auditory signals for spatial localization (see introduction; Ashmead et al., 1989; Kolarik et al., 2014). The presently used obstacles were small and thus could not have provided much information through echo localization. However, as this experiment was carried out in a medium-sized room, it is conceivable that blind subjects could use sound cues from reflections on the walls, helping them to have a better sense of position in the laboratory. To exclude this possibility, similar experiments with blind people wearing headphones could be performed.

Even if extravisual processing did not play a role it is conceivable that differences in spatial cognition are important. It could be expected that blindfolded people had an advantage in spatial cognition because of their previous visual experience; on the other hand it is possible that blind people are superior in spatial processing based on haptic information. Using the same amount of haptic exploration it is conceivable that blind people had learned to use such information more appropriately to locate the obstacles. For example, it was shown that late and congenitally blind participants are able to create accurate spatial mental images after locomotor learning of a full-scale navigable space, while blindfolded sighted participants are impaired in their 
ability to create precise spatial representations from locomotor experience (Afonso et al., 2010).

One of the main limitations in this study was the relatively small number of subjects. It is clear that more work on larger groups is needed before we will be able to fully understand these intriguing differences in obstacle crossing in blind subjects Nevertheless, despite these small numbers, some clear differences were already observed in the behavior of the leading limb. Details on the trailing limb have to await further study as well, since only the right leading limb was instrumented. It would be of interest to have data on both leading and trailing limb since it was previously shown that errors are more likely to occur for the trailing than for the leading limb (Heijnen et al., 2014). In future studies, measuring the two instrumented legs, it will also be possible to avoid limiting instructions (to cross with the right leg as leading limb).

Another limitation is that the measurements are restricted to the sagittal plane. In this case, other possible strategies, such as hip rotation, cannot be assessed in this work. Therefore, this is left for a future analysis with three-dimensional measurements.

With respect to future research, an interesting idea would be to include obstacle crossing with the expectation of a solid dangerous obstacle that resemble real-life situations and might elicit different responses.

The present results suggest that the lack of vision affects obstacle crossing differently depending on the duration of the vision loss (short-term vision loss in blindfolding vs. blindness). On the basis of the present data one would predict that recently acquired blindness would lead to a behavior more similar to the presently investigated blindfolded than to the long-term blind (congenitally blind). Long-term experience with lack of vision presumably increases confidence leading to less hesitant withholding behavior as observed in the blindfolded.

In conclusion, the present data provide evidence that blind people differ from blindfolded in their obstacle avoidance behavior (confirming our expectations). The data indicate that blind people cross obstacles more similarly to people with vision than to blindfolded subjects, although both groups showed increased foot clearance in the absence of vision. The blind group does this by adapting their obstacle crossing strategies in several gait parameters such as foot-obstacle distance at toe-off or hip-foot distance at foot contact, thereby becoming more similar to people with vision.

\section{ACKNOWLEDGMENTS}

Authors would like to thank the participants from Lar Escola Santa Luzia Para Cegos, Bauru, SP, Brazil.

\section{FUNDING}

V. D. Garcia thanks the Fundação de Amparo à Pesquisa do Estado de São Paulo, Brazil, for scholarship (FAPESP 06/06452-7). Dr. Forner-Cordero and Dr. Duysens acknowledge the National Research Council of Brazil for Research Grant (CNPq 311659/2012-8) and Visiting Professor Grant (CNPq 400819/2013-9), respectively.

\section{REFERENCES}

Afonso, A. L., Blum, A., Katz, B. F., Tarroux, P., Borst, G., \& Denis, M. (2010). Structural properties of spatial representations in blind people: Scanning images constructed from haptic exploration or from locomotion in a 3-D audio virtual environment. Memory \& Cognition, 38, 591-604. http://dx.doi.org/ 10.3758/MC.38.5.591

Ammons, C. H., Worchel, P., \& Dallenbach, K. M. (1953). Facial vision: the perception of obstacles out of doors by blindfolded and blindfolded-deafened subjects. The American Journal of Psychiatry, 66, 519-553.

Ashmead, D. H., Hill, E. W., \& Talor, C. R. (1989). Obstacle perception by congenitally blind children. Perception \& Psychophysics, 46, 425-433

Begg, R., Best, R., Dell'Oro, L., \& Taylor, S. (2007). Minimum foot clearance during walking: strategies for the minimisation of trip-related falls. Gait \& Posture, 25, 191-198

Byrne, J. M., \& Prentice, S. D. (2003). Swing phase kinetics and kinematics of knee replacement patients during obstacle avoidance. Gait \& Posture, 18, 95-104.

Chen, H. C., Ashton-Miller, J. A., Alexander, N. B., \& Schultz, A. B. (1991). Stepping over obstacles: gait patterns of healthy young and old adults. Journal of Gerontology, 46, M196-M203

Chou, L. S., \& Draganich, L. F. (1998). Placing the trailing foot closer to an obstacle reduces flexion of the hip, knee, and ankle to increase the risk of tripping. Journal of Biomechanics, 31, 685-91.

Elliott, D. B. (2014). The Glenn A. Fry award lecture 2013: blurred vision, spectacle correction, and falls in older adults. Optometry and Vision Science, 91, 593-601.

Erni, T., \& Dietz, V. (2001). Obstacle avoidance during human walking: learning rate and cross-modal transfer. The Journal of Physiology, 534, 303-312.

Eng, J. J., Winter, D. A., \& Patla, A. E. (1994). Strategies for recovery from a trip in early and late swing during human walking. Experimental Brain Research, 102, 339-349.

Forner-Cordero, A., Koopman, H. F. J. M., \& van der Helm, F. C. T. (2004). Mechanical model of the recovery from stumbling. Biological Cybernetics, 91, 212-222.

Forner-Cordero, A., Koopman, H. F. J. M., \& van der Helm, F. C. T. (2014). Mechanical model of the recovery reaction from stumbling: effect of step length on trunk control. Journal of the Brazilian Society of Mechanical, 36, 491-500.

Galna, B., Murphy, A. T., \& Morris, M. E. (2010). Obstacle crossing in people with Parkinson's disease: foot clearance and spatiotemporal deficits. Human Movement Science, 29, 843-852.

Hallemans, A., Ortibus, E., Meire, F., \& Aerts, P. (2010). Low vision affects dynamic stability of gait. Gait \& Posture, 32, 547-551.

Heasley, K., Buckley, J. G., Scally, A., Twigg, P., \& Elliott, D. B. (2004). Stepping up to a new level: effects of blurring vision in the elderly. Investigative Ophthalmology \& Visual Science, 45, 2122-2128

Heijnen, M. J., Muir, B. C., \& Rietdyk, S. (2012). Factors leading to obstacle contact during adaptive locomotion. Experimental Brain Research, 223, 219-231. 
Heijnen, M. J., Romine, N. L., Stumpf, D. M., \& Rietdyk, S. (2014). Memory-guided obstacle crossing: more failures were observed for the trail limb versus lead limb. Experimental Brain Research 232, 2131-2142.

Hof, A. L., Gazendam, M. G., \& Sinke, W. E. (2005). The condition for dynamic stability. Journal of Biomechanics, 38, 1-8.

Kolarik, A. J., Cirstea, S., Pardhan, S., \& Moore, B. C. (2014). A summary of research investigating echolocation abilities of blind and sighted humans. Hearing Research, 310, 60-8.

Lajoie, K., Bloomfield, L. W., Nelson, F. J., Suh, J. J., \& Marigold, D. S. (2012). The contribution of vision, proprioception, and efference copy in storing a neural representation for guiding trail leg trajectory over an obstacle. Journal of Neurophysiology, 107, 2283-2293.

Loverro, K. L., Mueske, N. M., \& Hamel, K. A. (2013). Location of minimum foot clearance on the shoe and with respect to the obstacle changes with locomotor task. Journal of Biomechanics, 46, 1842-1850.

Lu, T. W., Chen, S. C., \& Chiu, H. C. (2012). Best-compromise between mechanical energy expenditure and foot clearance predicts leading limb motion during obstacle-crossing. Gait \& Posture, 36, 552-556.

Marigold, D. S., \& Drew, T. (2011 May). Contribution of cells in the posterior parietal cortex to the planning of visually guided locomotion in the cat: effects of temporary visual interruption. Journal of Neurophysiology, 105, 2457-70.

McVea, D. A., \& Pearson, K. G. (2006). Long-lasting memories of obstacles guide leg movements in the walking cat. Journal of Neurophysiology, 26, 1175-1178.

McVea, D. A., \& Pearson, K. G. (2007). Stepping of the forelegs over obstacles establishes long-lasting memories in cats. Current Biology, 17, R621-R623.

McVea, D. A., Taylor, A. J., \& Pearson, K. G. (2009). Long-lasting working memories of obstacles established by foreleg stepping in walking cats require area, 5 of the posterior parietal cortex. The Journal of Neuroscience. 29, 9396-9404.

Mohagheghi, A. A., Moraes, R., \& Patla, A. E. (2004). The effects of distant and on-line visual information on the control of approach phase and step over an obstacle during locomotion. Experimental Brain Research, 155, 459-68.

Moraes, R., Lewis, M. A., \& Patla, A. E. (2004). Strategies and determinants for selection of alternate foot placement during human locomotion: influence of spatial and temporal constraints. Experimental Brain Research, 159, 1-13.

Muir, B. C., Haddad, J. M., Heijnen, M. J., \& Rietdyk, S. (2015). Proactive gait strategies to mitigate risk of obstacle contact are more prevalent with advancing age. Gait \& Posture, 41, 233-239.

Nakamura, T. (1997). Quantitative analysis of gait in the visually impaired. Disability and Rehabilitation, 19, 194-197.

Patla, A. E. (1998). How is human gait controlled by vision. Ecological Psychology, 10, 287-302.
Patla, A. E., Davies, T. C., \& Niechwiej, E. (2004). Obstacle avoidance during locomotion using haptic information in normally sighted humans. Experimental Brain Research, 155, 173185.

Patla, A. E., \& Greig, M. (2006). Any way you look at it, successful obstacle negotiation needs visually guided on-line foot placement regulation during the approach phase. Neuroscience Letters. 397, 110-114

Patla, A. E., \& Prentice, S. D. (1995). The role of active forces and intersegmental dynamics in the control of limb trajectory over obstacles during locomotion in humans. Experimental Brain Research, 106, 499-504.

Patla, A. E., \& Rietdyk, S. (1993). Visual control of limb trajectory over obstacles during locomotion: effect of obstacle height and width. Gait \& Posture, 1, 45-60.

Rhea, C. K., \& Rietdyk, S. (2007). Visual exteroceptive information provided during obstacle crossing did not modify the lower limb trajectory. Neuroscience Letters, 418, 60-65.

Rodrigues, S. T., Forner-Cordero, A., Garcia, V. D., Zago, P. F. P., \& Ferasoli, H. (2009). Influence of visual information on optimal obstacle crossing. In J. V., Sloten, P., Verdonck, M., Nyssen, J., Haueisen (Eds.), International federation for medical and biological engineering proceedings (Vol. 22, pp. 21332137) Berlin, Germany: Springer-Verlag.

Rossi, L. F., Rodrigues, S. T., \& Forner-Cordero, A. (2014, August). Do humans walk like robots when crossing an obstacle without visual information? Paper presented at the 5th IEEE RAS \& EMBS International Conference on Biomedical Robotics and Biomechatronics, Sáo Paulo, Brazil.

Santos, L. C., Moraes, R., \& Patla, A. E. (2010). Visual feedforward control in human locomotion during avoidance of obstacles that change size. Motor Control, 14, 424-439.

Schillings, A. M., Van Wezel, B. M., Mulder, T., \& Duysens, J. (1999). Widespread short-latency stretch reflexes and their modulation during stumbling over obstacles. Brain Research, 816, 480-486.

Strelow, E. R., \& Brabyn, J. A. (1982). Locomotion of the blind controlled by natural sound cues. Perception, 11, 635640.

Timmis, M. A., \& Buckley, J. G. (2012). Obstacle crossing during locomotion: visual exproprioceptive information is used in an online mode to update foot placement before the obstacle but not swing trajectory over it. Gait \& Posture, $36,160-162$.

Winnick, J. P. (2005). Adapted physical education and sport. Champaign, IL: Human Kinetics.

Received June 25, 2015

Revised October 2, 2015

Accepted December 5, 2015 